\title{
Healthcare Activity
}

National Cancer Institute

\section{Source}

National Cancer Institute. Healthcare Activity. NCI Thesaurus. Code C16205.

The actions of prevention, treatment, and management of illness and the preservation of mental and physical well-being through the services offered by the medical and allied health professions. 